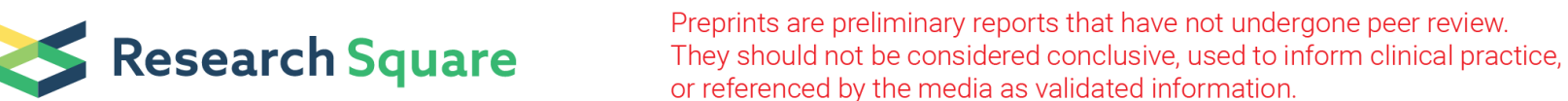

\section{The Impact of Rhodiola Rosea On Biomarkers of Diabetes, Inflammation, and Gut Microbiota in a Leptin Receptor-Knockout Mouse Model}

Mahtab Jafari ( $\nabla$ mjafari@uci.edu )

University of California, Irvine Jasmin Grace Juanson Arabit University of California, Irvine

Robert Courville

University of California, Irvine

Dara Kiani

University of Illinois at Chicago

John M. Chaston

Brigham Young University

Cindy Duy Nguyen

University of California, Irvine

Nilamani Jena

University of California, Irvine

Zhong-Ying Liu

University of California, Irvine

Prasanthi Tata

University of California, Irvine

Richard A. Etten

University of California, Irvine

\section{Research Article}

Keywords: diabetes, endocrine, disease, microbiota, pharmaceuticals

Posted Date: January 7th, 2022

DOI: https://doi.org/10.21203/rs.3.rs-1186409/v1

License: (1) (1) This work is licensed under a Creative Commons Attribution 4.0 International License.

Read Full License 


\section{Abstract}

Type 2 diabetes is the most prevalent endocrine disease in the world, and recently the gut microbiota have become a potential target for its management. Recent studies have illustrated that this disease may predispose individuals to certain microbiome compositions, and treatments like metformin have been shown to change gut microbiota and their associated metabolic pathways. However, given the limitations and side effects associated with pharmaceuticals currently being used for therapy of diabetes, there is a significant need for alternative treatments. In this study, we investigated the effects of a root extract from $R$ hodiola rosea in a Leptin receptor knockout $(d b / d b)$ mouse model of type 2 diabetes. Our previous work showed that Rhodiola rosea had anti-inflammatory and gut microbiome-modulating properties, while extending lifespan in several animal models. In this study, treatment with Rhodiola rosea improved the insulin response, and significantly decreased serum lipopolysaccharide and C-reactive protein levels. We hypothesize that these changes may in part reflect the modulation of the microbiota, resulting in improved gut barrier integrity and decreasing the translocation of inflammatory biomolecules into the bloodstream. These findings indicate that Rhodiola rosea is an attractive candidate for further research in the management of type 2 diabetes.

\section{Introduction}

Type 2 diabetes (T2D) is a metabolic disease that currently ranks as one of the largest concerns of global public health, affecting an estimated 476 million people worldwide ${ }^{1}$. With both genetic and environmental factors contributing to this complex disease, diabetes is a leading cause of mortality in many countries and globally impacts life expectancy in both developed and developing nations ${ }^{1,2}$. One of the hallmarks of the disease is hyperglycemia resulting from defects in insulin secretion, insulin action, or both ${ }^{3}$. Of the three forms of clinical diabetes, the vast majority (about $90 \%$ ) of patients have T2D, which is characterized by insulin resistance ${ }^{3}$. Whereas there are many environmental and behavioral factors that modulate genetic susceptibility to this form of diabetes, the prevalence of T2D has risen steadily in recent decades $^{4}$. Due to rising health costs associated with the increasing incidence and prevalence of diabetes worldwide ${ }^{5,6}$, identifying and evaluating safe and cost-effective therapeutic interventions in the management of T2D is of increasing importance. Current treatment algorithms for T2D include lifestyle changes, as well as oral and parenteral drugs ${ }^{7}$. However, many current treatments have significant limitations or side effects that can impact this large patient population. Although metformin is first-line treatment for T2D, it has side effects including nausea and diarrhea and is contraindicated in patients predisposed to lactic acidosis ${ }^{7,8}$. Insulin secretagogues such as sulfonylureas and meglitinides tend to lose their efficacy over time due to beta cell failure ${ }^{9,10}$, while alpha-glucosidase inhibitors, thiazolidinediones, and dipeptidyl peptidase- 4 inhibitors have unique mechanisms of action, but adverse effects such as weight gain, heart failure, and gastrointestinal issues have led to poor compliance with therapy ${ }^{11-14}$. Thus, there is a need for new therapeutic interventions in T2D that are safe and effective. 
There is significant evidence that inflammation plays a central role in the pathogenesis of T2D through two distinct pathways ${ }^{15}$. Obesity, specifically visceral adiposity, causes systemic inflammation through infiltration of adipose tissue by macrophages ${ }^{16,17}$ and production of pro-inflammatory cytokines such as TNF- $a, \mathrm{IL}-1 \beta, \mathrm{IL}-6$, and CCL2 ${ }^{18,19}$, which act as antagonists of insulin signaling ${ }^{20}$. Recent studies also suggest that differences in the human gut microbiome between normal and diabetic subjects ${ }^{21}$ are linked to systemic inflammation through altered gut integrity 22,23 , increased circulating gram-negative bacteria and endotoxin ${ }^{22,24}$, as well as lipopolysaccharide (LPS)-induced inflammatory cytokine secretion through TLR-4 signaling ${ }^{24,25}$. Evidence in humans also suggests that certain gut microbiome compositions may predispose individuals to conditions such as obesity and diabetes ${ }^{26,27}$. As crucial components that moderate host health and physiology, the gut microbiome can contribute to inflammation, alterations of intestinal linings, dyslipidemia, and a wide variety of other changes ${ }^{23}$. However, studies also indicate that modulation of the gut microbiome can decrease LPS-induced systemic inflammation in a mouse T2D model, thereby counteracting these changes ${ }^{25}$. Together, these observations suggest that targeting inflammation through the microbiome could be a novel approach to treating $T 2 D^{17,28}$.

Plant-derived therapeutics such as cinnamon and curcumin extracts have been shown to have potential anti-diabetic properties, although their efficacy has not been evaluated in randomized clinical trials ${ }^{29,30}$. The adaptogenic plant Rhodiola rosea is used as a medicinal in traditional medical practices worldwide and has been shown to have anti-inflammatory and gut microbiome-modulating properties ${ }^{31-33}$. As an adaptogen, $R$. rosea represents an important category of pharmacological substances that are known to aid the body in resisting a wide variety of stressors (i.e. biological, chemical, physical, etc.) to maintain homeostasis and stabilize physiological processes that may be disrupted ${ }^{32}$. R. rosea extract appears to be safe in human studies ${ }^{34-36}$, which makes it an attractive candidate for the treatment of T2D. $R$. rosea extended lifespan in several animal models including worms, snails, and flies ${ }^{37-40}$. When tested on a fly model deficient in the insulin receptor substrate chico, $R$. rosea still extended lifespan but decreased expression levels of Drosophila insulin-like peptide (dILP) 2, 3, and 5 in wild-type flies ${ }^{39}$, suggesting complex effects on the insulin signaling pathway. However, the effects of this plant extract on an animal model of diabetes have not yet been evaluated.

Here, we investigated the effects of a root extract from $R$. rosea in a Leptin receptor knockout $(d b / d b)$ mouse model of T2D. Leptin is a key adipokine responsible for maintenance of energy homeostasis and body mass, whereas mice lacking the Leptin receptor display hyperphagia and consequently develop obesity, visceral adiposity, hyperglycemia, and hyperinsulinemia ${ }^{41-43}$. In other mice models, deficiencies in Leptin as well as beta-cell dysfunction have been linked to T2D, suggesting that this adipokine plays a key role in the pathogenesis of diabetes ${ }^{44}$. Like human diabetics, $d b / d b$ mice show augmented expression of the differentiation marker Aldh $1 \mathrm{a} 3$ and reduced nuclear expression of the transcription factor Nkxx 6.1, and exhibit hyperglycemia compared to age-matched non $d b / d b$ mouse model ${ }^{45}$. These observations make the $d b / d b$ mouse model suitable for the goals of this study. 


\section{Results}

Rhodiola rosea improves glucose homeostasis in diabetic $\mathrm{db} / \mathrm{db}$ mice

To assess the effects of Rhodiola rosea on a mouse model of T2D, we treated a cohort of 6 week-old leptin receptor-knockout $(d b / d b)$ mice with an extract of $R$. rosea that was verified for quality based on validated biomarkers (see Methods), administered daily by oral gavage for 4 weeks. Control mice received gavage with water. After 4 weeks, both R. rosea-treated and control $d b / d b$ mice developed fasting hyperglycemia, and there were no significant differences between the cohorts in response to an intraparenteral glucose challenge (glucose tolerance test; Supplementary Fig. 1). Following a week of recovery, we next tested the response of the treatment cohorts to an exogenous insulin challenge (insulin tolerance test) at T5, one week after supplementation had ended. At this time point, the fasting blood glucose levels of the two cohorts were significantly different, with the control gavage group displaying higher levels (Fig. 1). R. rosea-treated mice showed a response to insulin challenge, with decreasing blood glucose over a 2 hour period post-injection, while control mice lost their response to insulin after 30 minutes. This suggests that $R$. rosea treatment improves the insulin resistance that is characteristic of $d b / d b$ mice.

\section{Rhodiola rosea does not affect the body mass of $\mathrm{db} / \mathrm{db}$ mice}

We evaluated the body mass of the mice on a weekly basis during the study to assess whether potential changes in blood glucose levels of the $R$. rosea supplemented mice and the controls were due to a decrease in obesity. Although the mean body weight of $R$. rosea-treated $d b / d b$ mice was lower than control water-treated mice in weeks 8 through 14 of the study, these differences were not statistically significant (Fig. 2).

\section{Rhodiola rosea modestly modulates the gut microbiome of $\mathrm{db} / \mathrm{db}$ mice}

To assess the effect of $R$. rosea on the gut microbiome of $d b / d b$ mice, we collected serial stool samples (pretreatment and weeks 1,2, and 6 on treatment) from females of the two cohorts and analyzed the composition of the microbial community of the gut with $16 \mathrm{~S}$ rRNA amplicon next-generation sequencing. The microbiome of both cohorts were dominated by Bacteroidetes and Firmicutes, and most of the reads could be designated as amplicon sequence variants (ASVs) within four major taxonomic assignments: the families Rikenellaceae and S24-7 (Bacteroidetes), and the family Lachnospiraceae and genus Lactobacillus (Firmicutes). Of time and $R$. rosea treatment, time accounted for the most variation in the microbiota composition of water and $R$. rosea-treated mice (Fig. 3). When the microbiota composition of the two cohorts was subjected to Bray-Curtis analysis ${ }^{46}$, which does not account for phylogenetic relationships, there was a significant effect of $R$. rosea treatment on the microbiome of $d b / d b$ mice (Fig. 4 and Table 1). The only taxa that varied with treatment or with time and treatment were reads assigned to the Desulfovibrionales, clustered at the order level. These were more abundant in $R$. rosea-treated mice than control mice (Fig. 5), including during the time point before $R$. rosea was administered, suggesting this difference may be more strongly attributed to differences in microbiota composition before the experiment began (e.g. early cage effects) than to $R$. rosea treatment. Together, these findings identify 
modest but significant variation in the composition of the mouse gut microbiota associated with $R$. rosea treatment.

\section{Table 1}

Results of PERMANOVA on Bray-Curtis distances of microbiota data.

\begin{tabular}{|lllllll|}
\hline & Degrees of freedom & Sum of Squares & Mean Squares & $\mathbf{f}$ & $\mathbf{R}^{2}$ & p-value \\
\hline Treatment & 1 & 0.2 & 0.2 & 3.64 & 0.04 & 0.02 \\
\hline Time Point & 3 & 2.89 & 0.96 & 17.69 & 0.55 & 0 \\
\hline Interaction & 3 & 0.35 & 0.12 & 2.14 & 0.07 & 0.02 \\
\hline Residuals & 34 & 1.85 & 0.05 & $\mathrm{NA}$ & 0.35 & $\mathrm{NA}$ \\
\hline Total & 41 & 5.29 & $\mathrm{NA}$ & $\mathrm{NA}$ & 1 & $\mathrm{NA}$ \\
\hline
\end{tabular}

Rhodiola rosea decreases circulating lipopolysaccharide levels and pro-inflammatory cytokines

To observe whether the changes of the gut microbiota associated with $R$. rosea treatment had any physiological consequences, we determined the LPS content from serum samples from the two cohorts via a limulus amoebocyte lysate ( $L A L)$ assay. Treatment with $R$. rosea decreased the lipopolysaccharide (LPS) levels in the serum by almost 50\% (Fig. 5). Whereas circulating LPS (derived from gram-negative bacteria) triggers production of inflammatory cytokines by tissue macrophages and other cells, to further observe inflammation levels exhibited by the $d b / d b$ mice, we determined if $R$. rosea treatment was associated with changes in C-reactive protein (CRP), an inflammatory marker exclusively produced by hepatocytes. Similar to LPS, $R$. rosea treatment decreased hepatic CRP transcript expression by about $40 \%$ (Fig. 6).

\section{Discussion}

The goal of this study was to evaluate the impact of $R$. rosea, a medicinal plant of emerging interest and possible therapeutic value, on the phenotype and the gut microbiota of the $d b / d b$ mouse model of T2D. Although this study shares a common format with a number of published microbiota-disease interaction articles $^{47-50}$, it is one of the few studies that evaluates the impact of a botanical extract on the gut microbiota throughout the progression of T2D. Although the format of our study was correlative and associative, the results may serve as the basis for future mechanism-based studies.

We elected to use a model with a severe phenotype of diabetes, $d b / d b$ mice, because we wanted to investigate whether $R$. rosea can improve glucose homeostasis in a T2D model that is similar to severe and advanced human T2D in terms of visceral adiposity and insulin resistance. Due to an absence of leptin receptor expression in the brain, the $d b / d b$ mice consume excess calories and develop obesity and insulin resistance, exhibiting hyperglycemia and dyslipidemia ${ }^{43}$. The use of this severe model may have prevented us from observing beneficial effects of $R$. rosea that might be relevant to less severe 
phenotypes of diabetes (i.e., pre-diabetes), but allowed us to study the effects of $R$. rosea in a T2D mouse model without resorting to a high-fat diet or other dietary manipulations.

We observed no significant differences between the two cohorts in fasting blood glucose or in the response to a parenteral glucose challenge (GTT) after 4 weeks of treatment, which is unsurprising given that $d b / d b$ mice have extreme fasting hyperglycemia $(>800 \mathrm{mg} / \mathrm{dL})$ at 10 weeks of age ${ }^{45}$ (Supplementary Fig. 1). However, at T5 (one week after treatment had ended), R. rosea significantly lowered the fasting blood glucose of $d b / d b$ mice. This suggests that more prolonged administration of $R$. rosea may modulate the T2D phenotype, either by improving the function of insulin-responsive tissues in $d b / d b$ mice or ameliorating pancreatic beta cell exhaustion that is observed in this model ${ }^{45}$. The $R$. roseatreated mice also had significantly lower blood glucose levels in response to insulin challenge, suggesting that the extract improves insulin sensitivity in the $d b / d b$ model.

To rule out that the decreases in blood glucose levels were a direct effect of $R$. rosea and not a consequence of decreased food intake and reduction in obesity, we measured the body mass of the $d b / d b$ mice over time. Since there were no significant differences in the body mass, we conclude that $R$. rosea did not affect the hyperphagia associated with the $d b / d b$ phenotype, an observation consistent with other studies demonstrating that $R$. rosea prolongs lifespan through a mechanism distinct from caloric restriction ${ }^{47}$. Therefore, it is plausible that $R$. rosea improves glucose homeostasis in $d b / d b$ mice through other mechanisms.

It has been reported previously that $d b / d b$ mice displays elevated levels of bacteria in the gut from the S24-7 family compared to wild-type populations ${ }^{51}$, and that result was observed here, with S24-7 bacteria dominating the composition of the microbiome in both cohorts at all time points. Additionally, the presence of sulfate-reducing bacterial species from the Desulfovibrionales order has been associated with the pathology of T2D 21 . In this study, the only reads that varied across treatments were the reads from the order Desulfovibrionales. However, we were surprised to see higher relative abundance of these bacteria in the $R$. rosea treatment group than in the control group, since it has previously been reported that treatment with salidroside, a glycoside found in $R$. rosea, causes a decrease in Desulfovibrionales levels ${ }^{52}$. Within the group of mice that received $R$. rosea treatments, the abundance of Desulfovibrionales were more abundant than in controls during the first two timepoints, but had comparable abundances between the control and $R$. rosea treatments at timepoints T2 and T6. We cannot explain why the two populations differed at the T0 timepoint, which preceded any treatment, but we observed two possible patterns that are not mutually exclusive. First, the decrease in relative abundance of Desulfovibrionales from T0 to T2 in the $R$. rosea treatment suggests the treatment may affect bacterial abundance. Second, the convergence of Desulfovibrionales abundance in the $R$. rosea-treated and control cohorts at the T2 and $T 6$ time points suggests we may be observing a change in abundance that is determined by shared factors between the groups, such as changes in age.

Multiple studies have demonstrated major changes in the gut microbiota during the development of obesity and T2D in humans ${ }^{27,53,54}$. When compared to healthy control groups, humans with T2D 
exhibited significantly reduced proportions of phylum Firmicutes and class Clostridia, in addition to compositional changes in the microbiota ${ }^{53,54}$. These changes may provide a protective advantage to mice that received $R$. rosea as seen by a reduction in downstream inflammatory markers (Fig. 5). It has been reported that the gut integrity depends on the gut microbiota and that the modulation of the gut microbiota may lower intestinal permeability by a mechanism involving glucagon-like peptide-2 (GLP$2)^{19}$. Gut integrity plays an important role in the whole-body homeostasis since its disruption causes metabolic endotoxemia, which is the translocation of LPS from Gram-negative bacteria into the bloodstream from the gut, and has been linked to chronic inflammation ${ }^{16}$. Our group has shown that $R$. rosea is not directly influencing the microbial composition through any direct anti-microbial activity ${ }^{33}$. In our previous study, the plant extract did not show anti-microbial effects when tested on bacteria isolated from Drosophila melanogaster whole-body homogenate ${ }^{33}$.

In humans, excess calories have been shown to play a role in the induction of changes to the gut bacterial community ${ }^{55}$. According to a recent report, $d b / d b$ mice display impaired gut barrier integrity compared to wild-type non-diabetic mice ${ }^{56}$. It has also been shown that in mice, high-fat diet-induced microbial dysbiosis led to a decrease in gut integrity and controlled metabolic endotoxemia ${ }^{25}$. Due to the close relationship between the gut microbiota and gut integrity ${ }^{22,25}$, we evaluated the LPS levels in the serum of the $d b / d b$ mice. We observed that mice on water gavage had significantly higher levels of LPS in serum compared to the $R$. rosea-treated mice. We postulate that the modulation of the gut microbiota by the plant extract improved gut barrier integrity, thus lowering the rate of translocation of LPS into the bloodstream, which in turn, decreased expression of a hepatic inflammatory marker.

Analysis of the mentioned data led us to postulate that $R$. rosea improved insulin sensitivity of $d b / d b$ mice by modest modulation of the gut microbiota which decreased metabolic endotoxemia and subsequent inflammation. The improvements in fasting blood glucose levels and insulin response may be due to the decreased inflammatory status of the host wherein normal cell processes may have been prioritized instead of stress responses ${ }^{15}$.

In conclusion, while previous studies have illustrated that $R$. rosea has anti-inflammatory and gut microbiome-modulating properties which have been reported to extend lifespan in several animal models, the findings of this study present a possible mechanism of action for the plant extract and its effects on a diabetic mouse model. This is important, in part, because the Leptin receptor knockout $(d b / d b)$ mouse model of T2D that was analyzed is based on genetic characteristics-which eliminated the vast array of environmental and behavioral factors that otherwise may have acted as confounding variables. While only a modest modulation of the gut microbiome was indicated in this study, we hypothesize that these changes may have improved the integrity of the gut barrier, thereby accounting for the significant decreases seen in both serum lipopolysaccharide and hepatic $\mathrm{C}$-reactive protein levels. These findings suggest that $R$. rosea is a good candidate for further investigation as a potential treatment for T2D. However, additional research is needed to elucidate if the beneficial effects of $R$. rosea are mediated 
through modulation of intestinal epithelial cell integrity, resulting in normal intestinal permeability in the diabetic environment.

\section{Methods}

All methods were performed in accordance with applicable guidelines and regulations.

\section{Mice}

BKS.Cg-Dock $7^{m+/+} L e p r^{d b} / \mathrm{J}$ and C57BI/6 mice were obtained from the Jackson Laboratory (Bar Harbor, $\mathrm{ME})$. $L e p r^{d b} / \mathrm{J}$ mice were bred to generate homozygous $L e p r^{d b} / L e p r^{d b}(d b / d b)$ pups which were used to model T2D. Mice were maintained in 12 hour light/dark and had free access to food and water. Mice were weaned when they were 3 weeks old, and entered into the study at 6 weeks of age.

\section{Study Timeline}

The study timeline is summarized in Supplementary Fig. 2. Mice were administered either water (control) or $125 \mathrm{mg} / \mathrm{kg} R$. rosea extract (treatment) by oral gavage daily for the 4 weeks of treatment, initiated when the mice were 6 weeks old (T0) and continued until the mice were 10 weeks old (T4). The quality of the $R$. rosea extract was verified by HPLC that showed the extract contained $1.3 \%$ salidroside and $3.9 \%$ rosavins, consistent with a high-quality extract (Data on File). Fecal samples were collected at 6 weeks of age (T0, prior to treatment), 7 weeks of age (T1), 8 weeks of age (T2) and 12 weeks of age (T6). The Glucose Tolerance Test (GTT) was performed at week 10 and Insulin Tolerance Test (ITT) was performed at week 11. Mice were sacrificed and dissected at Week 14. The study was approved by the Instituitional Animal Care and Use Committee (IACUC) of UCl. All experiments were performed in accordance with UCI IACUC guidelines and regulations and with ARRIVE guidelines (https://arriveguidelines.org).

\section{Glucose Tolerance Test (GTT) and Insulin Tolerance Test (ITT)}

Given the importance of in vivo mouse models for studying the pathogenesis of T2D and various treatment interventions, several methods have been developed to investigate glucose tolerance, as well as the secretion and action of insulin in these models ${ }^{57}$. To ascertain potential differences between treatment and control groups, with regards to glucose homeostasis, both glucose and insulin tolerance tests were performed. Four hours before the assays were performed, the food was removed to induce a state of fasting. Blood samples $(\sim 2 \mu \mathrm{L})$ were drawn from the mouse tail to record the blood glucose level using an AlphaTrak2 Blood Glucose Monitoring System. The first drop of blood from the mouse tail was wiped away, and the second drop was used to record values. Blood glucose values were recorded 30, 60, and 90 minutes after the injection of either $0.5 \mathrm{~g} / \mathrm{kg}$ interperotionel of $10 \%$ glucose (BW $\times 5 \mu \mathrm{L}$ ) or 0.75 $\mathrm{IU} / \mathrm{kg}$ ip of $0.25 \mathrm{IU} / \mathrm{mL}$ insulin (BW x 3). Data were analyzed using GraphPad Prism 8.

\section{Microbiome analysis}


Fecal samples from the mice were collected at 6 weeks of age (T0, prior to treatment), 7 weeks of age (T1), 8 weeks of age (T2) and 12 weeks of age (T6). DNA was extracted by the initiative using the Zymobiomics Mini DNA kit. 16S rRNA amplicon PCR was performed, targeting the V4-V5region using the EMP primers ( $515 \mathrm{~F}$ (barcoded) and $926 \mathrm{R})^{58}$. The samples were prepared into a library that was sequenced at the UC Irvine Genomics High Throughput Facility on an Illumina MiSeq, using paired-end 300 bp v3 sequencing chemistry. The raw sequence data were imported into QIIME2 (qiime2.org) ${ }^{59,60}$ and demultiplexed ${ }^{58}$. This bioinformatics platform has recently been rewritten and reengineered for the next generation of microbiome sequencing, facilitating taxanomic and phylogenetic analyses. While dozens of software packages written in various programming languages are often needed for comprehensive analyses of this type of sequencing data, QIIME2 allows for "sequence quality checking, denoising, taxonomic classification, alignment, and phylogenetic tree building"-allowing for seamless analysis, description, and quantification of microbial communities ${ }^{58}$.

The sequences were assigned a taxonomic classification using the q2-feature-classifier ${ }^{61}$ on a GreenGenes database downloaded in July 2020 from the QIIME2 website (qiime2.org) ${ }^{62}$. Because the quality scores of the data were low, no read pairs passed DADA2 quality filter steps ${ }^{63}$. Therefore, we analyzed the data only using the forward reads, which had higher average quality scores than the reverse reads. Forward reads that passed quality filtering and denoising by default DADA2 parameters in QIIME2 were rarefied to 11,390 reads per sample and beta-diversity distance metrics were calculated using QIIME2 ${ }^{64,65,46}$. As part of calculating the Unifrac beta-diversity metrics we constructed a phylogenetic tree with fasttree $2^{66}$ based on mafft alignment ${ }^{67}$. PERMANOVA ${ }^{68}$ and $\mathrm{ANCOM}^{69}$ analyses were performed in $\mathrm{R}$.

\section{LAL assay of blood serum}

Serum samples were diluted 40 -fold and were evaluated using the Pierce ${ }^{\mathrm{TM}}$ Chromogenic Endotoxin Quant Kit according to the manufacturer's recommended protocol. Data were analyzed using GraphPad Prism 8.

\section{Body Mass Assay}

The body mass of mice from both treatments was assayed from weeks 8-14 to test for obesity related differences. Data from male and female subjects were pooled, and Mann-Whitney tests at each timepoint indicated there were no significant differences in body weights. Data were analyzed using GraphPad Prism 8.

\section{Gene Expression Assay}

RNA was extracted from the liver tissue using Trizol. The RNA extract was treated with DNA-free ${ }^{\mathrm{TM}}$ DNA Removal Kit from Invitrogen to remove any contaminating DNA. A cDNA library was generated with the iScript ${ }^{\mathrm{TM}}$ CDNA Synthesis Kit from Bio-Rad. qPCR was performed on a BioRadMJ Mini Personal Thermal Cycler with iQ SYBR Green Supermix. The PCR amplification program consisted of an initial denaturation 
set at $94^{\circ} \mathrm{C}$ for 3 minutes, followed by 40 three-step cycles at $94^{\circ} \mathrm{C}$ for 10 seconds, $60^{\circ} \mathrm{C}$ at 30 seconds and at $72^{\circ} \mathrm{C}$ for 45 seconds. The $\Delta \Delta \mathrm{Ct}$ Method was used to quantify the relative expression of genes of interest ${ }^{46}$. The CRP (gene of interest) and GAPDH (reference gene) primers were derived from PrimerBank (Supplementary Table 1). Data were analyzed using GraphPad Prism 8.

\section{Declarations}

\section{ACKNOWLEDGMENTS}

We would like to acknowledge Ms. Claudia Weihe, Dr. Jennifer Martiny and Dr. Katrine Whiteson of the Microbiome Initiative at UC Irvine for their advice and work on the 16s rRNA analysis of the microbiome of the $d b / d b$ mice. We would like to thank UCI School of Medicine-Pharmaceutical Scienes Collaborative Research Funds and Mr. John P Wareham and Mrs. Lois C. Wareham for providing funding for this work.

\section{AUTHOR CONTRIBUTIONS}

\section{Mahtab Jafari}

As the primary investigator, Dr. Jafari designed and performed experiments and oversaw the data analysis (except for the microbiome data analysis). She also wrote the manuscript.

Jasmin Grace Juanson Arabit

As an undergraduate student in Jafari Lab, Ms. Arabit participated in the experiments and coordinated the work.

\section{Robert Courville}

As a graduate student in Jafari Lab, Mr. Courville was involved in the microbiome data analysis, revising sections of the manuscript, and reference management.

\section{Dara Kiani}

As an undergraduate student in Jafari Lab, Mr. Kiani was involved in collecting and handlging the microbiome samples and make recommendations on the microbiome samples data analysis.

\section{John M. Chaston}

As a research collaborator, Dr. Chaston analyzed the microbiome data, developed the graphs and participated extensively in writing the results and discussion on micriobiome data.

\section{Cindy Duy Nguyen}

As an undergraduate student in Jafari Lab, Ms. Nguen participated in two of the mouse experiments. 


\section{Nilamani Jena}

As a project scientis in Dr. Van Etten lab, Dr. Jena, particiapted in maintaining the mouse colonies and in mouse experiments (injections, blood draws, etc.)

\section{Zhong-Ying Liu}

As the lab manager in Dr. Van Etten lab, Dr. Liu, particiapted in maintaining the mouse colonies and in mouse experiments (injections, blood draws, etc.)

\section{Prasanthi Tata}

As a research assistant in Dr. Van Etten lab, Ms. Tata, particiapted in mouse experiments (injections, blood draws, etc.) and provided feedback.

\section{Richard A. Van Etten ${ }^{4}$}

As a research collaborator, Dr. Van Etten helped designed the mouse experiments and provided scientific advice on all the experiments. He also participated in revising and finalizing the manuscript.

\section{COMPETING INTERESTS}

The author(s) declare no competing interests.

\section{References}

1. Lin, X. et al. Global, regional, and national burden and trend of diabetes in 195 countries and territories: an analysis from 1990 to 2025. Scientific reports 10, 1-11 (2020).

2. Mambiya, M. et al. The Play of Genes and Non-genetic Factors on Type 2 Diabetes. Front Public Health 7, 349 (2019).

3. American Diabetes Association. Diagnosis and classification of diabetes mellitus. Diabetes care 33, S62-S69 (2010).

4. Murea, M., Ma, L. \& Freedman, B. I. Genetic and environmental factors associated with type 2 diabetes and diabetic vascular complications. Rev Diabet Stud 9, 6-22 (2012).

5. Smyth, S. \& Heron, A. Diabetes and obesity: the twin epidemics. Nature medicine 12, 75-80 (2006).

6. American Diabetes Association. Economic costs of diabetes in the US in 2017. Diabetes care 41, 917-928 (2018).

7. Marín-Peñalver, J. J., Martín-Timón, l., Sevillano-Collantes, C. \& del Cañizo-Gómez, F. J. Update on the treatment of type 2 diabetes mellitus. World journal of diabetes 7, 354 (2016).

8. Lee, H. \& Ko, G. Effect of Metformin on Metabolic Improvement and Gut Microbiota. Applied and Environmental Microbiology 80, 5935-5943 (2014). 
9. Takahashi, A. et al. Sulfonylurea and glinide reduce insulin content, functional expression of KATP channels, and accelerate apoptotic $\beta$-cell death in the chronic phase. Diabetes research and clinical practice $77,343-350$ (2007).

10. Maedler, K. et al. Sulfonylurea induced $\beta$-cell apoptosis in cultured human islets. The Journal of Clinical Endocrinology \& Metabolism 90, 501-506 (2005).

11. Chiasson, J.-L. et al. Acarbose treatment and the risk of cardiovascular disease and hypertension in patients with impaired glucose tolerance: the STOP-NIDDM trial. Jama 290, 486-494 (2003).

12. Abe, M., Okada, K. \& Soma, M. Antidiabetic agents in patients with chronic kidney disease and endstage renal disease on dialysis: metabolism and clinical practice. Current drug metabolism 12, 5769 (2011).

13. Bogacka, I., Xie, H., Bray, G. A. \& Smith, S. R. The effect of pioglitazone on peroxisome proliferatoractivated receptor-y target genes related to lipid storage in vivo. Diabetes care 27, 1660-1667 (2004).

14. Rosenwasser, R. F., Sultan, S., Sutton, D., Choksi, R. \& Epstein, B. J. SGLT-2 inhibitors and their potential in the treatment of diabetes. Diabetes, metabolic syndrome and obesity: targets and therapy 6, 453 (2013).

15. Gregor, M. F. \& Hotamisligil, G. S. Inflammatory mechanisms in obesity. Annual review of immunology 29, 415-445 (2011).

16. Sweet, M. J. \& Hume, D. A. Endotoxin signal transduction in macrophages. Journal of leukocyte biology 60, 8-26 (1996).

17. $\mathrm{Xu}, \mathrm{H}$. et al. Chronic inflammation in fat plays a crucial role in the development of obesity-related insulin resistance. The Journal of clinical investigation 112, 1821-1830 (2003).

18. Shoelson, S. E., Lee, J. \& Goldfine, A. B. Inflammation and insulin resistance. The Journal of clinical investigation 116, 1793-1801 (2006).

19. Berg, A. H. \& Scherer, P. E. Adipose tissue, inflammation, and cardiovascular disease. Circulation research 96, 939-949 (2005).

20. Peraldi, P., Hotamisligil, G. S., Buurman, W. A., White, M. F. \& Spiegelman, B. M. Tumor necrosis factor (TNF)-a inhibits insulin signaling through stimulation of the p55 TNF receptor and activation of sphingomyelinase. Journal of Biological Chemistry 271, 13018-13022 (1996).

21. Qin, J. et al. A metagenome-wide association study of gut microbiota in type 2 diabetes. Nature 490, 55-60 (2012).

22. Cani, P. D. et al. Changes in gut microbiota control inflammation in obese mice through a mechanism involving GLP-2-driven improvement of gut permeability. Gut 58, 1091-1103 (2009).

23. Kirschman, L. J. \& Milligan-Myhre, K. C. The Costs of Living Together: Immune Responses to the Microbiota and Chronic Gut Inflammation. Applied and Environmental Microbiology 85, e02147-18.

24. Cani, P. D. et al. Metabolic endotoxemia initiates obesity and insulin resistance. Diabetes 56, 17611772 (2007). 
25. Cani, P. D. et al. Changes in gut microbiota control metabolic endotoxemia-induced inflammation in high-fat diet-induced obesity and diabetes in mice. Diabetes 57, 1470-1481 (2008).

26. Musso, G., Gambino, R. \& Cassader, M. Interactions between gut microbiota and host metabolism predisposing to obesity and diabetes. Annual review of medicine 62, 361-380 (2011).

27. Kalliomäki, M., Carmen Collado, M., Salminen, S. \& Isolauri, E. Early differences in fecal microbiota composition in children may predict overweight. The American journal of clinical nutrition $\mathbf{8 7}, \mathbf{5 3 4 -}$ 538 (2008).

28. Fantuzzi, G. Adipose tissue, adipokines, and inflammation. Journal of Allergy and clinical immunology 115, 911-919 (2005).

29. Chuengsamarn, S., Rattanamongkolgul, S., Luechapudiporn, R., Phisalaphong, C. \& Jirawatnotai, S. Curcumin extract for prevention of type 2 diabetes. Diabetes care 35, 2121-2127 (2012).

30. Kirkham, S., Akilen, R., Sharma, S. \& Tsiami, A. The potential of cinnamon to reduce blood glucose levels in patients with type 2 diabetes and insulin resistance. Diabetes, obesity and metabolism 11, 1100-1113 (2009).

31. Lee, Y. et al. Anti-inflammatory and neuroprotective effects of constituents isolated from Rhodiola rosea. Evidence-based complementary and alternative medicine 2013, (2013).

32. Bawa, A. \& Khanum, F. Anti-inflammatory activity of Rhodiola rosea-"a second-generation adaptogen". Phytotherapy Research: An International Journal Devoted to Pharmacological and Toxicological Evaluation of Natural Product Derivatives 23, 1099-1102 (2009).

33. Labachyan, K. E., Kiani, D., Sevrioukov, E. A., Schriner, S. E. \& Jafari, M. The impact of Rhodiola rosea on the gut microbial community of Drosophila melanogaster. Gut pathogens 10, 1-10 (2018).

34. Darbinyan, V. et al. Clinical trial of Rhodiola rosea L. extract SHR-5 in the treatment of mild to moderate depression. Nordic journal of psychiatry 61, 343-348 (2007).

35. Olsson, E. M., von Schéele, B. \& Panossian, A. G. A randomised, double-blind, placebo-controlled, parallel-group study of the standardised extract shr- 5 of the roots of Rhodiola rosea in the treatment of subjects with stress-related fatigue. Planta medica 75, 105-112 (2009).

36. Shevtsov, V. et al. A randomized trial of two different doses of a SHR-5 Rhodiola rosea extract versus placebo and control of capacity for mental work. Phytomedicine 10, 95-105 (2003).

37. Chen, C. et al. Rhodiola rosea extends lifespan and improves stress tolerance in silkworm, Bombyx mori. Biogerontology 17, 373-381 (2016).

38. Wiegant, F. A. C. et al. Plant adaptogens increase lifespan and stress resistance in C. elegans. Biogerontology 10, 27-42 (2009).

39. Schriner, S. E. et al. Extension of Drosophila lifespan by Rhodiola rosea through a mechanism independent from dietary restriction. PloS one 8, e63886 (2013).

40. Boon-Niermeijer, E. K., Van den Berg, A., Wikman, G. \& Wiegant, F. A. C. Phyto-adaptogens protect against environmental stress-induced death of embryos from the freshwater snail Lymnaea stagnalis. Phytomedicine 7, 389-399 (2000). 
41. Tartaglia, L. A. et al. Identification and expression cloning of a leptin receptor, OB-R. Cel/ 83, 12631271 (1995).

42. Bray, G. A. \& York, D. A. Hypothalamic and genetic obesity in experimental animals: an autonomic and endocrine hypothesis. Physiological reviews 59, 719-809 (1979).

43. Kobayashi, K. et al. The $\mathrm{db} / \mathrm{db}$ mouse, a model for diabetic dyslipidemia: molecular characterization and effects of Western diet feeding. Metabolism 49, 22-31 (2000).

44. Chen, W. S. et al. Leptin Deficiency and Beta-Cell Dysfunction Underlie Type 2 Diabetes in Compound Akt Knockout Mice. Molecular and Cellular Biology 29, 3151-3162 (2009).

45. Burke, S. J. et al. db/db mice exhibit features of human type 2 diabetes that are not present in weight-matched C57BL/6J mice fed a western diet. Journal of diabetes research 2017, (2017).

46. Sorensen TA. A method of establishing groups of equal amplitude in plant sociology based on similarity of species content and its application to analyses of the vegetation on Danish commons. Biol. Skar.. 1948;5:1-34.

47. Membrez, M. et al. Gut microbiota modulation with norfloxacin and ampicillin enhances glucose tolerance in mice. The FASEB Journal 22, 2416-2426 (2008).

48. Chou, C. J., Membrez, M. \& Blancher, F. Gut decontamination with norfloxacin and ampicillin enhances insulin sensitivity in mice. Personalized Nutrition for the Diverse Needs of Infants and Children 62, 127-140 (2008).

49. Geurts, L. et al. Altered gut microbiota and endocannabinoid system tone in obese and diabetic leptin-resistant mice: impact on apelin regulation in adipose tissue. Frontiers in microbiology 2, 149 (2011).

50. Liu, W. et al. Grape seed proanthocyanidin extract ameliorates inflammation and adiposity by modulating gut microbiota in high-fat diet mice. Molecular nutrition \& food research 61, 1601082 (2017).

51. Yu, F. et al. Abnormal gut microbiota composition contributes to the development of type 2 diabetes mellitus in db/db mice. Aging (Albany NY) 11, 10454 (2019).

52. Yuan, Y., Wu, X., Zhang, X., Hong, Y. \& Yan, H. Ameliorative effect of salidroside from Rhodiola Rosea L. on the gut microbiota subject to furan-induced liver injury in a mouse model. Food and Chemical Toxicology 125, 333-340 (2019).

53. Wu, X. et al. Molecular characterisation of the faecal microbiota in patients with type II diabetes. Current microbiology 61, 69-78 (2010).

54. Larsen, N. et al. Gut microbiota in human adults with type 2 diabetes differs from non-diabetic adults. PloS one 5, e9085 (2010).

55. Jumpertz, R. et al. Energy-balance studies reveal associations between gut microbes, caloric load, and nutrient absorption in humans. The American journal of clinical nutrition 94, 58-65 (2011).

56. Brun, P. et al. Increased intestinal permeability in obese mice: new evidence in the pathogenesis of nonalcoholic steatohepatitis. American Journal of Physiology-Gastrointestinal and Liver Physiology 
292, G518-G525 (2007).

57. Beguinot, F. \& Nigro, C. Measurement of glucose homeostasis in vivo: glucose and insulin tolerance tests. in Animal Models in Diabetes Research 219-228 (Humana Press, 2012).

58. Hall, M. \& Beiko, R. G. 16S rRNA gene analysis with QIIME2. in Microbiome analysis 113-129 (Springer, 2018).

59. Bolyen, E. et al. Reproducible, interactive, scalable and extensible microbiome data science using QIIME 2. Nature biotechnology 37, 852-857 (2019).

60. Whelan FJ, Rossi L, Stearns JC, Surette MG. 2018. Culture and molecular profiling of the respiratory tract microbiota, p. 49-61. In Microbiome Analysis. Springer.

61. Bokulich NA, Kaehler B, Rideout JR, Dillon MR, Bolyen E, Knight R, Huttley GA, Caporoso JG. 2018. Optimizing taxonomic classification of marker-gene amplicon sequences with QIIME 2's q2-featureclassifier plugin. Microbiome, 6(1) 90.

62. McDonald D, Price MN, Goodrich J, Nawrocki EP, Desantis TZ, Probst A, Andersen GL, Knight R, Hugenholtz P. 2012. An improved Greengenes taxonomy with explicit ranks for ecological and evolutionary analyses of bacteria and archaea. The ISME journal, 6(3), 610-618.

63. Callahan BJ, McMurdie PJ, Rosen MJ, Han AW, Johnson AJ, Holmes SP. DADA2: High-resolution sample inference from Illumina amplicon data. Nat Methods. 2016;13(7):581-583.

64. Lozupone CA, Hamady M, Kelley ST, Knight R. Quantitative and qualitative beta diversity measures lead to different insights into factors that structure microbial communities. App/ Environ Microbiol. 2007;73(5):1576-1585.

65. Lozupone C, Knight R. UniFrac: a new phylogenetic method for comparing microbial communities. Appl Environ Microbiol. 2005;71(12):8228-8235.

66. Price MN, Dehal PS, Arkin AP. FastTree 2--approximately maximum-likelihood trees for large alignments. PLoS One. 2010;5(3):e9490.

67. Katoh K, Misawa K, Kuma K, Miyata T. MAFFT: a novel method for rapid multiple sequence alignment based on fast Fourier transform. Nucleic Acids Res. 2002;30(14):3059-3066.

68. Oksanen J, Blanchet F, Friendly M, Kindt R, Legendre P, McGlinn D, Minchin P, O'Hara R, Simpson G, Solymos P, Stevens M, Szoecs E, Wagner H. 2020. vegan: Community Ecology Package.

69. Mandal S, Van Treuren W, White RA, Eggesbø M, Knight R, Peddada SD. 2015. Analysis of composition of microbiomes: a novel method for studying microbial composition. Microbial ecology in health and disease 26:27663.

\section{Figures}




\section{ITT}

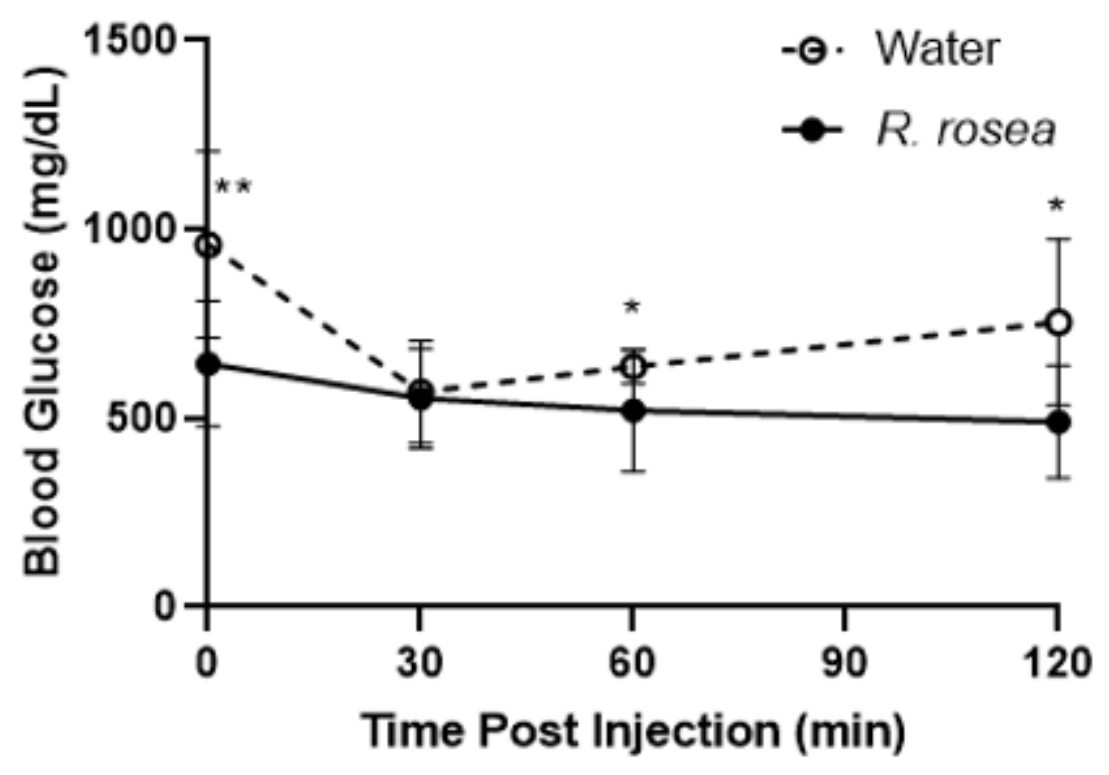

Figure 1

Improved insulin tolerance in $R$. rosea-treated $d b / d b$ mice. Insulin tolerance test of $d b / d b$ mice. ${ }^{*} P<0.05$, $\star * P<0.01$, multiple t-tests; $\mathrm{n}=7$ for water and $\mathrm{n}=11$ for $R$. rosea.

\section{Body Mass}

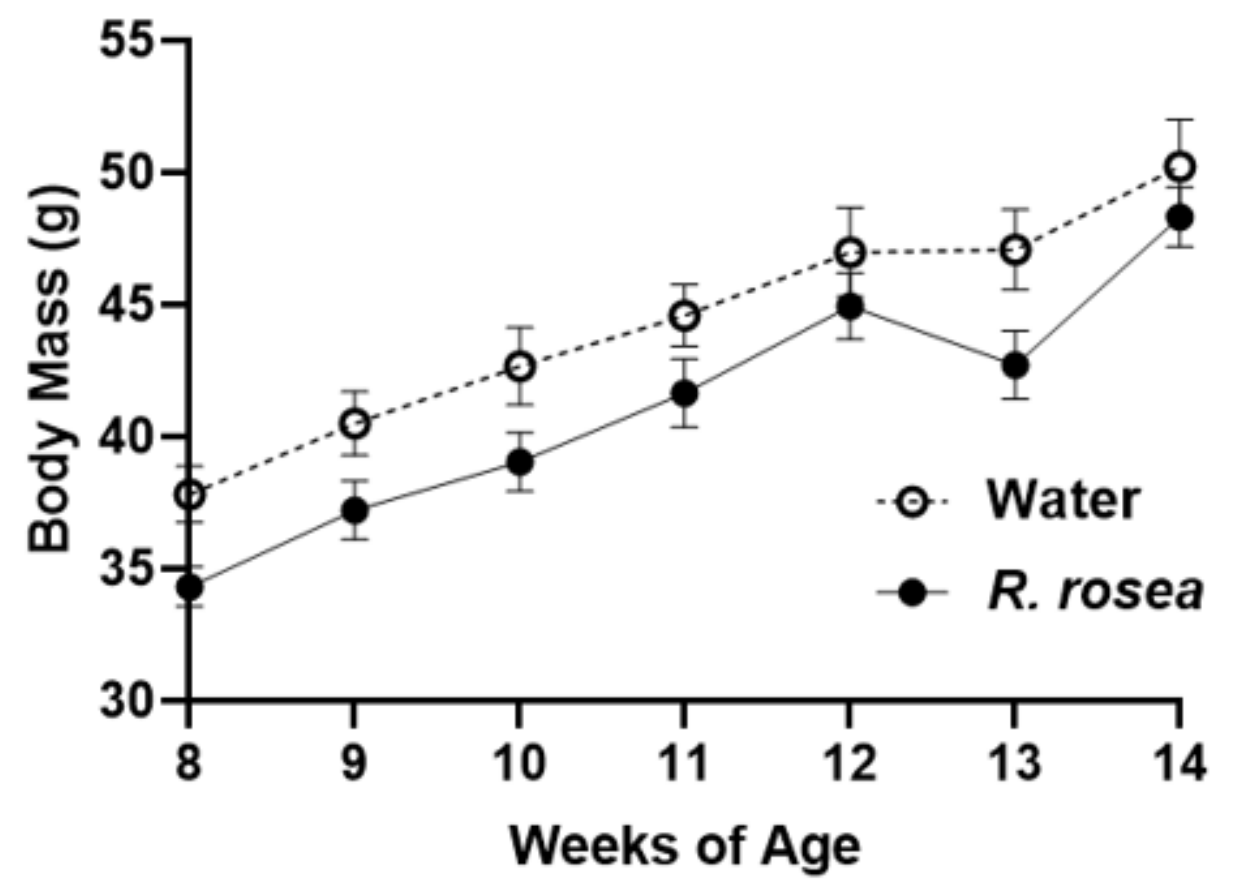

Figure 2 
No effect of $R$. rosea on body weight of $d b / d b$ mice. Body mass measurements of $d b / d b$ mice. No significant differences were found (Mann-Whitney tests at each time point, $\mathrm{n}=7$ for water and $\mathrm{n}=11$ for $R$. rosea treatment).

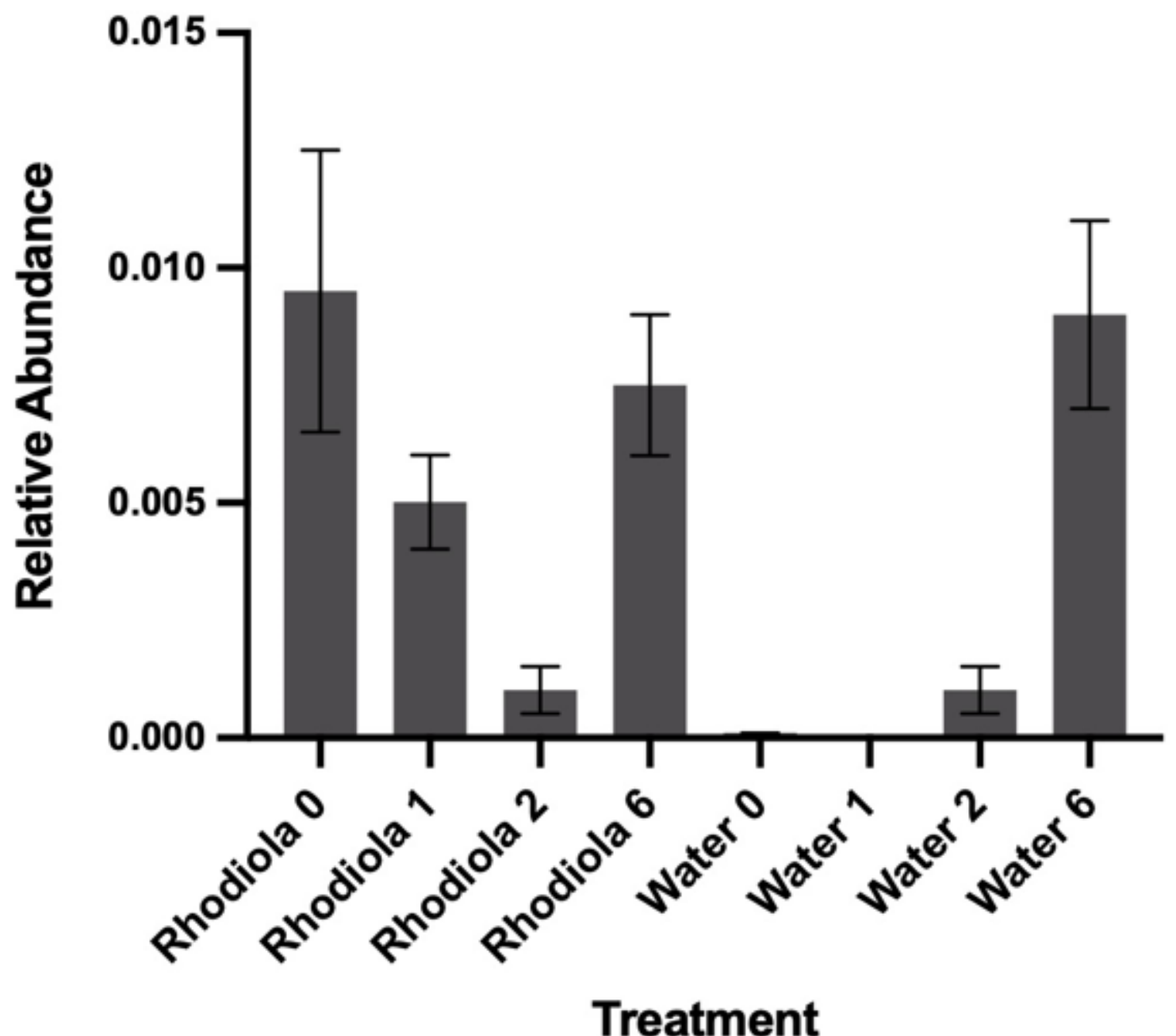

Figure 3

Effects of $R$. rosea on the gut microbiome of $d b / d b$ mice. A) Analysis of abundance shows composition is dominated by bacteria from the Bacteroidetes phylum, especially from the S24-7 family. B) Bray-Curtis principal coordinate ordination. The corresponding PERMANOVA (Table 1) identifies significant differences in microbiota composition with treatment and time. 


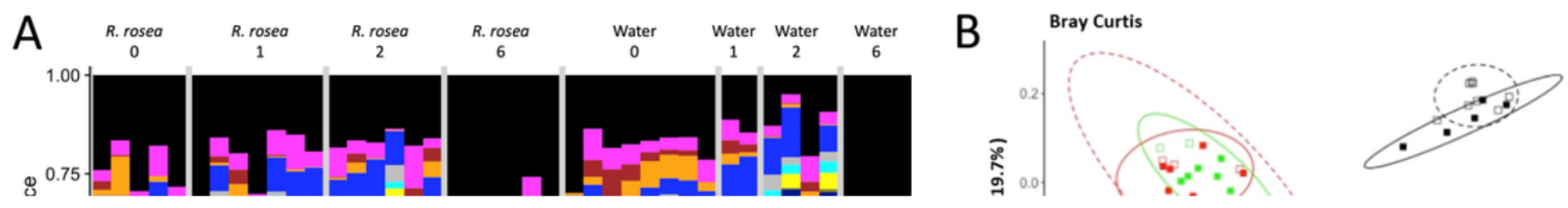

Figure 4

Treatment differences in fractional abundance of bacteria from the Desulfovibrionales order at each timepoint sampled. Higher abundances were observed in mice treated with $R$. rosea than the control at pretreatment and one week following treatment. No statistically significant differences were observed at the $\mathrm{T} 2$ and $\mathrm{T} 6$ timepoints.

\section{Serum LPS Concentration}

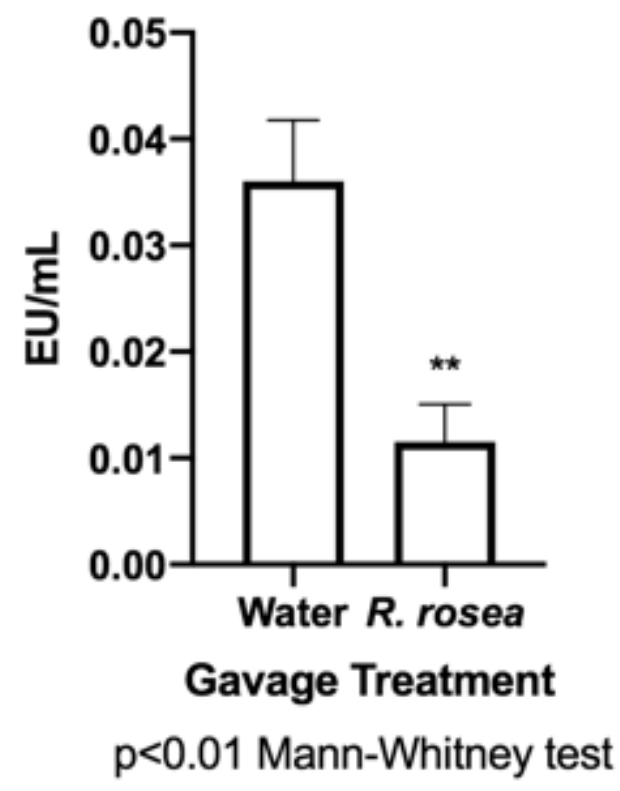

Figure 5

$R$. rosea treatment reduces circulating LPS. LAL assay of mouse serum. $P<0.01$, Mann-Whiney test; $\mathrm{n}=8$ for water and $\mathrm{n}=6$ for $R$. rosea. 


\section{Liver CRP Gene Expression}

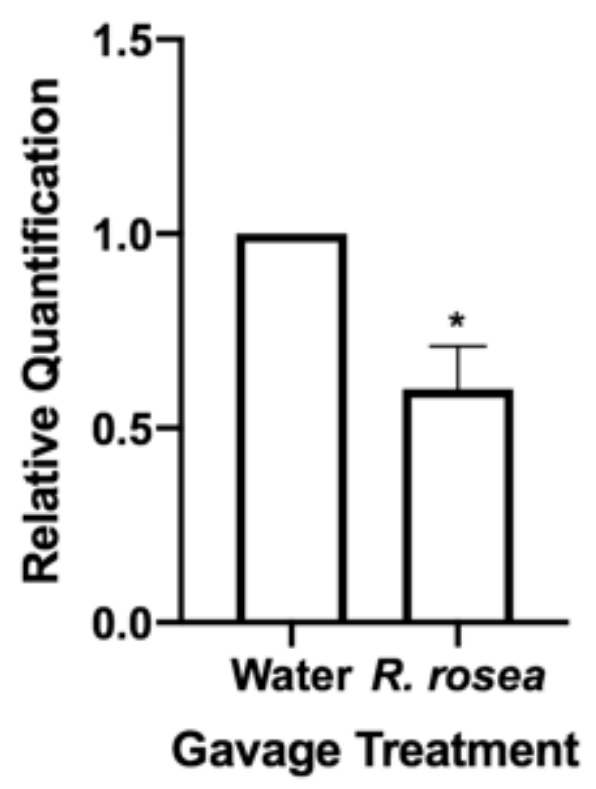

$\mathrm{p}<0.05$

One sample t and Wilcoxon test

Figure 6

$R$. rosea decreases hepatocyte CRP expression. Relative expression of CRP transcripts in mouse liver calculated using the DDCt method. $\mathrm{n}=8$ for water and $\mathrm{n}=6$ for $R$. rosea, ${ }^{\star} P<0.05$.

\section{Supplementary Files}

This is a list of supplementary files associated with this preprint. Click to download.

- RhodiolaMicrobiomeLeptingSupplementaryInofrmation122921.docx 